\title{
Aphasic status epilepticus as the sole symptom of epilepsy: A case report and literature review
}

\author{
JI-QING QIU $^{1 *}$, YU CUI $^{2 *}$, LI-CHAO SUN ${ }^{3}$ and ZHAN-PENG ZHU ${ }^{1}$ \\ Departments of ${ }^{1}$ Neurosurgery, ${ }^{2}$ Otolaryngology and ${ }^{3}$ Emergency, \\ The First Hospital of Jilin University, Changchun, Jilin 130021, P.R. China
}

Received October 14, 2016; Accepted June 2, 2017

DOI: $10.3892 /$ etm.2017.4979

\begin{abstract}
Aphasia is a common symptom encountered by neurologists. However, the presence of aphasia as the sole manifestation of partial status epilepticus is rare. The present study reports a case of aphasic status epilepticus in a 27-year-old right-handed female who presented after the abrupt onset of aphasia, which had persisted for 1.5 days. The patient's medical history included head trauma followed by a craniectomy and cranioplasty. Computed tomography scans revealed a lesion in the patient's left parietal lobe, and an electroencephalogram showed a spike and slow wave pattern in the left hemisphere of the brain during aphasia. The patient's condition improved after the oral administration of oxcarbazepine daily. In the present study it was observed that EEGs were a simple method to diagnose aphasic seizures and therefore EEG recordings should be performed in all cases of unexplained aphasia. In addition, the present study reviewed previously reported cases of aphasic status epilepticus.
\end{abstract}

\section{Introduction}

Aphasia is an acquired neurological disorder that impairs the ability of patients to communicate (1). Aphasia can be classified into Broca's aphasia, Wernicke's aphasia and global aphasia (1). Different types of aphasia may be caused by lesions in different regions of the brain (2). Ictal or postictal aphasia, also known as epileptic aphasia, occurs following epileptic seizures and is a common event (3). Aphasia may resolved with the end of epilepsy. However, de novo aphasia that appears as

Correspondence to: Dr Zhan-Peng Zhu, Department of Neurosurgery, The First Hospital of Jilin University, 71 Xinmin Street, Changchun, Jilin 130021, P.R. China

E-mail: 282324491@qq.com

${ }^{*}$ Contributed equally

Abbreviations: CT, computed tomography; AED, anti-epileptic drug; EEG, electroencephalogram

Key words: aphasia status epilepticus, epilepsy, EEG the sole clinical manifestation of status epilepticus is rare and often short in duration (4,5). Aphasic status epilepticus may be rapidly reversed by using antiepileptic drugs (4).

The present study reports a unique case of aphasic status epilepticus as the sole symptom of epilepsy in an adult woman. The patient had experienced a previous left parietal lobe trauma and subsequent craniectomy. Furthermore, the current study presents a literature review of articles on epileptic aphasia.

\section{Case report}

A 27-year-old right-handed woman was admitted to emergency room of the Department of Neurosurgery, The First Hospital of Jilin University (Changchun, China). The patient's family detailed that the patient had suffered from a sudden regression in their language abilities that had persisted for the past 1.5 days. The patient could follow verbal commands, but had lost the ability to talk. There was no known history of epileptic seizures in the patient or their family. The patient had no history of any known abnormalities in the buccofacial muscle. However, at the age of 19 , the patient had suffered from a left parietal lobe trauma that led to a intracranial hematoma in the left parietal lobe. The patient was left in a coma. To relieve the internal pressure and aid recovery, the patient underwent a decompressive craniectomy and, at the age of 22, the patient received a cranioplasty to repair their skull.

Upon admission, a computed tomography (CT) scan performed in the emergency room revealed a lesion in the left parietal lobe (Fig. 1). A complete neurological examination lead to the patient being diagnosed with Broca's aphasia without any additional symptoms. The results of a routine blood panel and lumbar puncture were unremarkable. However, an electroencephalogram (EEG) revealed an abnormal spike and slow wave pattern in the central-parietal-temporal region of the left hemisphere of the brain (Fig. 2).

The patient was capable of initiating and performing normal behavioral actions, and following nonverbal commands, during the basic neurological examination and EEG recording. Furthermore, the patient was able to recall events that occurred during the ictal phase and thus neuropsychological evaluation of higher cortical functions was not required.

Following the diagnosis of aphasic status epilepticus, the patient was treated with $10 \mathrm{mg}$ diazepam administered 

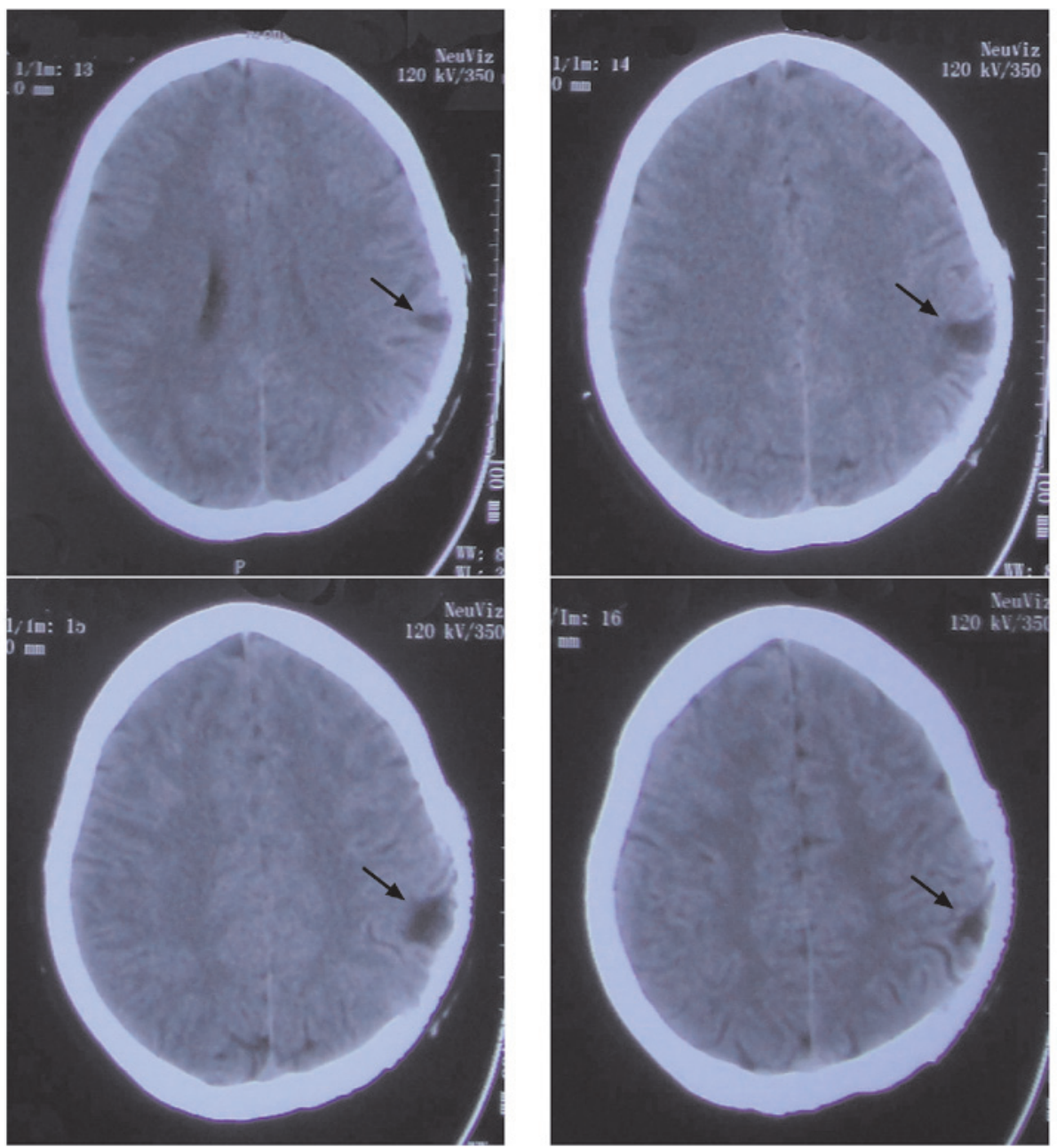

Figure 1. Computed tomography scans performed on the 27-year-old female patient upon admission, which revealed a lesion on the left parietal lobe. Four different slices of the cerebral lesion are shown. The arrow indicates the lesion.

intravenously over 2 min, which was repeated 10 min later. EEG demonstrated a rapid reversal of the aphasic disorder following diazepam treatment (Fig. 3). The patient was discharged. The following day, aphasia developed again and so the patient was orally administered $300 \mathrm{mg}$ oxcarbazepine daily for 1 week and then $450 \mathrm{mg}$ daily the second week; however, no clinical improvement was observed. Therefore, in the third week, the oral oxcarbazepine dosage was increased to $600 \mathrm{mg}$ daily, after which the aphasic symptoms significantly improved. The patient continued to receive $600 \mathrm{mg}$ oxcarbazepine daily long-term. The present report was approved by the Ethics Committee of The First Hospital of Jilin University and the patient provided written informed consent.

\section{Literature review}

Aphasic status epilepticus is a rare condition. It has previously been described in a few reports, which are summarized in Table I. The PubMed (www.ncbi.nlm.nih.gov/pubmed) and Web of Science (www.webofknowledge.com) were searched independently by two reviewers, using key words 'aphasia status epilepticus', 'aphasia' and 'status epilepticus'. As of August 2016, 23 cases of aphasic status epilepticus have been reported, 13 in men and 10 in women, suggesting a tendency for male predominance. The patient in the present study was 27 years old at the onset of aphasia. Only 1 previously reported patient with aphasic status epilepticus (6) was younger than the patient in the present study. The cause of aphasic status epilepticus in the previously published reports included stroke, multiple sclerosis or other diseases. Among the 23 cases, 6 cases were Broca's aphasia (4,6-10), 7 were Wernicke's aphasia (9,11-16) and 10 cases were global aphasia $(5,17-25)$. The presence of prior seizures was reported in 5/23 cases. CT/magnetic resonance imaging findings in the left hemisphere of the brain were reported in $15 / 23$ cases. A total of $8 / 23$ patients $(34.8 \%)$ presented with aphasia following a stroke $(4,7,9,11,13,17,18,22)$, which is in accordance with data published in a previous report (26). A total of 6 patients exhibited rapid improvement after the administration of anti-epileptic drugs (AEDs) $(6,8,11,13,19,22)$. Because diazepam and phenytoin are broad-spectrum AEDs, they are commonly used for the treatment aphasic status epilepticus $(4-6,8,9,17)$. However, since oxcarbazepine is an AED for the treatment of complex partial seizures, it was used to treat the patient in the present report. Wells et al (5) reported a case of global aphasic status epilepticus in a patient presenting with glioblastoma 

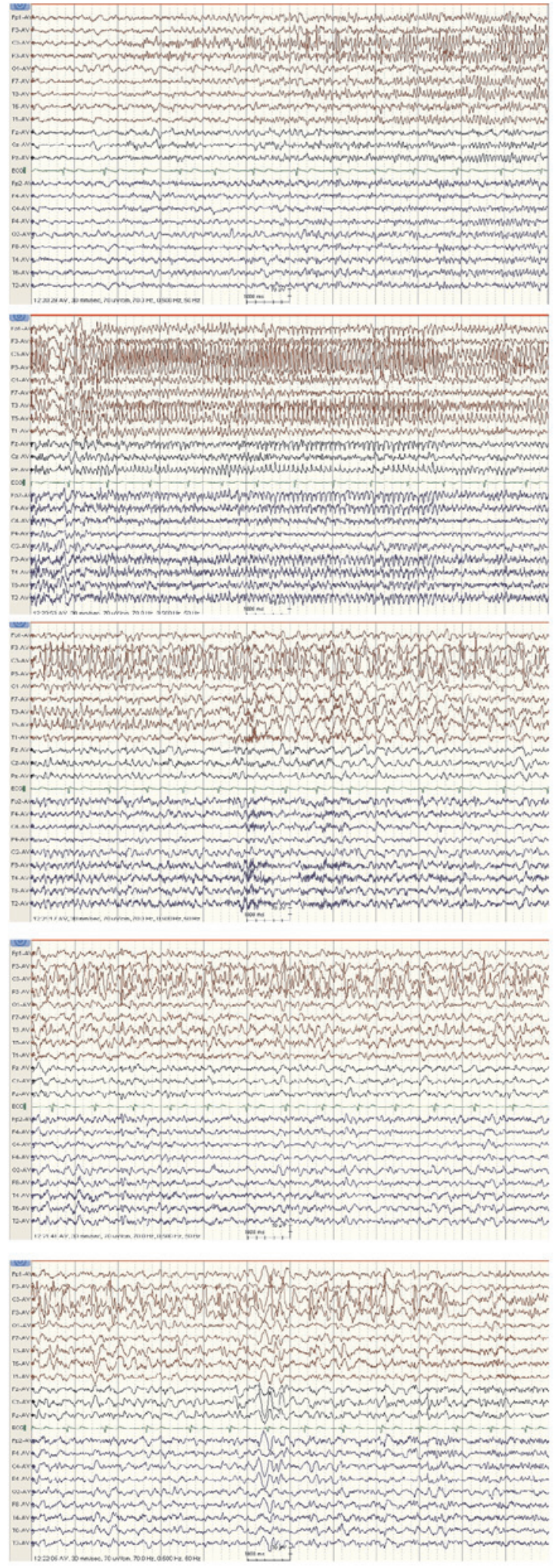
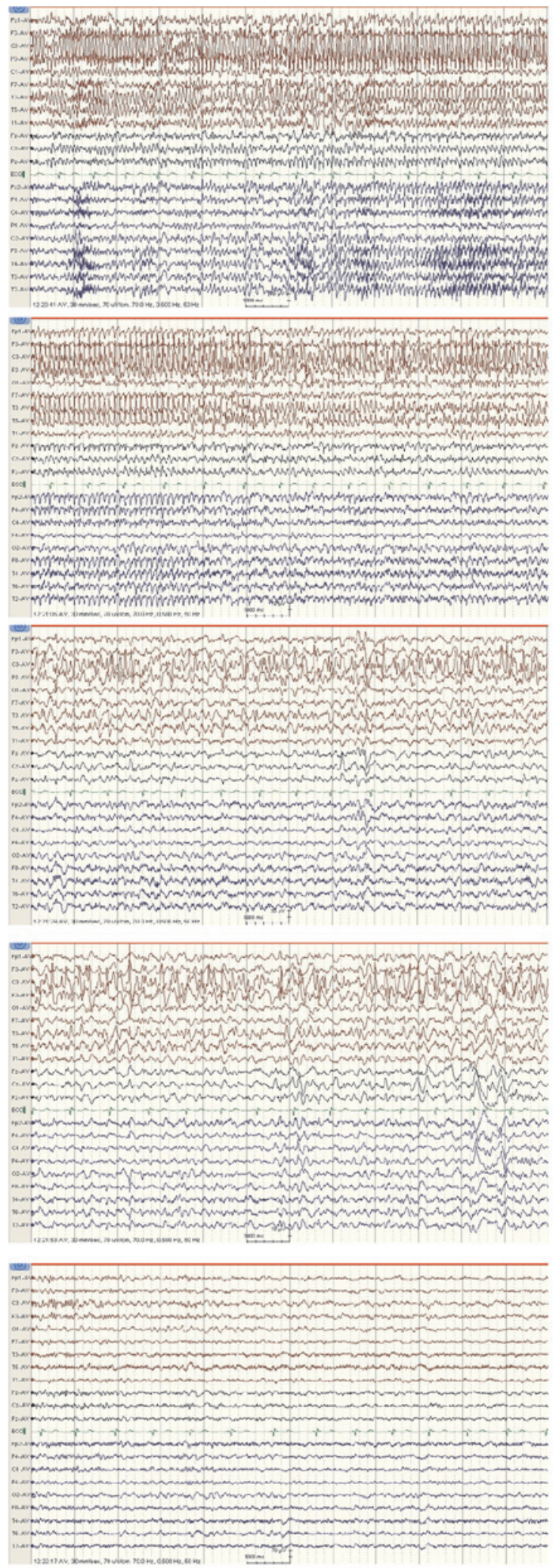

Figure 2. EEGs of the seizure ictal performed on the 27-year-old female patient upon the admission. The EEG revealed irregular spikes and slow wave delta activity in the left central and temporal regions, leading to a diagnosis of Broca's aphasia. EEG recorded the change of wave during the ictal phase. EEG, electroencephalogram.

multiforme. Racy et al (9) reported aphasia in a patient presenting with a cranial defect in their left parieto-occipital area. In the present case report, the patient had a traumatic lesion in their left parietal lobe.

\section{Discussion}

Aphasia as an exclusive symptom of epilepsy is rare. The diagnostic criteria for aphasic seizures were first introduced 


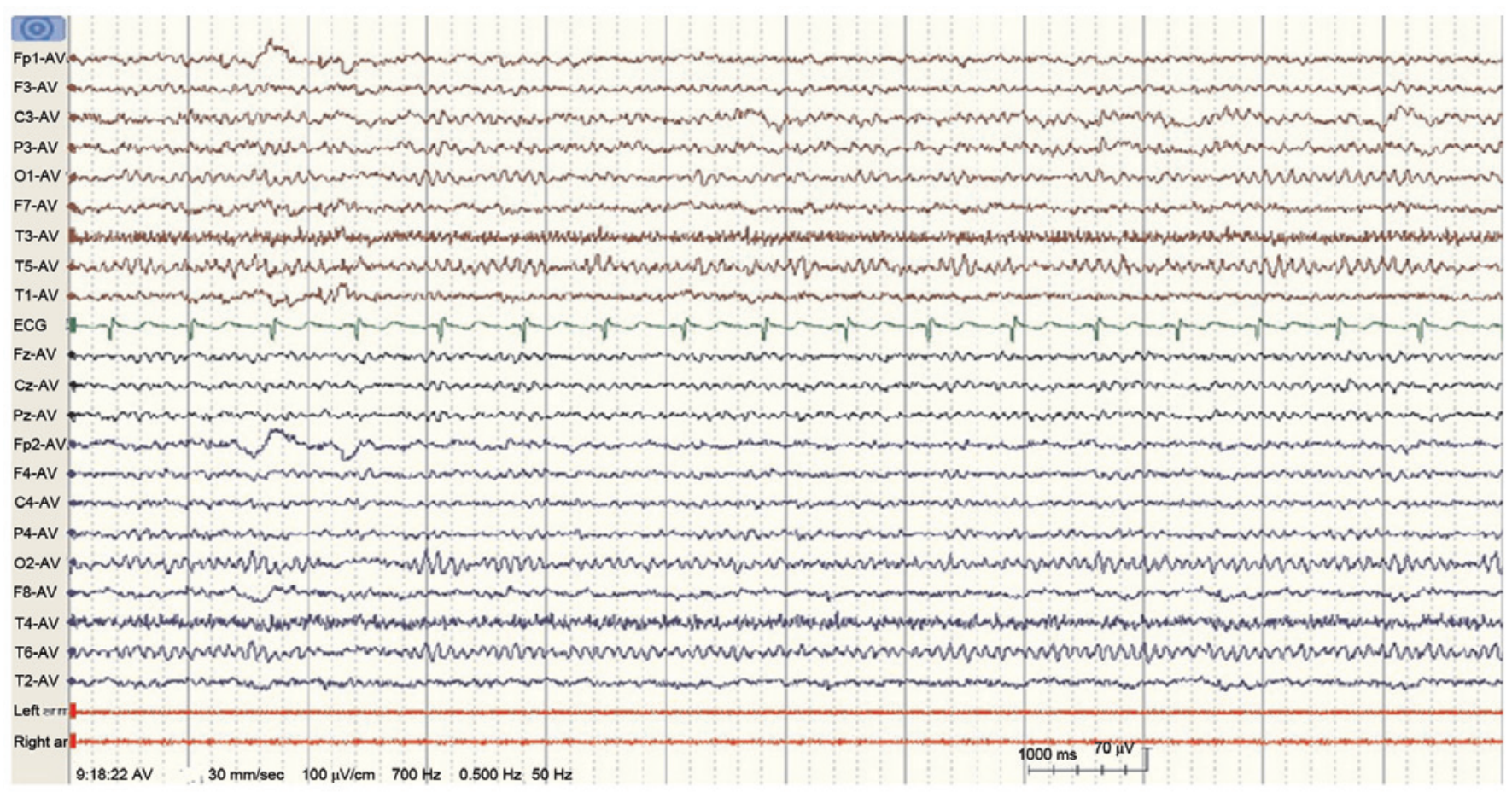

Figure 3. Electroencephalogram performed after the administration of antiepileptic treatment demonstrated the reversal of the aphasic disorder.

by Rosenbaum et al (7) in 1986 and then modified by Grimes and Guberman (4) in 1997. The criteria include the preservation of language production during seizures followed by a post-ictal aphasic phase with the maintenance of consciousness. Furthermore, the aphasia should be associated with seizures, as documented by EEG, and improve following the successful treatment of the seizures $(4,7)$. In the present report, the case of a 27 -year old female who fulfilled the above-mentioned criteria for aphasic seizures was presented. The patient presented with sudden aphasia without any known abnormalities to their buccofacial muscle or a history of epileptic seizures. The patient's family detailed that the patient could talk and communicate normally prior to the onset of aphasia. An EEG recording of seizure activity was the primary method for the accurate diagnosis of ictal aphasia in the present case. Therefore, an EEG should be performed for all patients that present with sudden aphasia without other clinical symptoms.

Acquired epileptic aphasia, also known as Landau-Kleffner syndrome, is a rare neurological disorder characterized by severe and prolonged receptive language deterioration, with patients suffering from total loss of auditory/verbal comprehension and expression. Patients typically present with additional behavioral disorders, including hyperactivity, inattentiveness or withdrawal (27). Acquired epileptic aphasia generally affects children between the ages of 3 and 7 years old. In the present study, the patient was 27 years old, and experienced sudden onset aphasia without prior auditory or verbal comprehension and expression defects; therefore, acquired epileptic aphasia was excluded from the differential diagnosis.

Cerebrovascular diseases are the leading cause of aphasia; however, aphasia can also occur secondary to traumatic, epileptic or neurodegenerative conditions associated with critical language areas of the brain (26). Furthermore, it has previously been reported that trauma and cranioplasty are risk factor for post-operative seizures (28). The differential diagnoses for the patient in the present study included stroke, other structural or metabolic damage and inflammation. The present study used a CT scan to exclude stroke, which revealed a lesion in the left parietal lobe. Lumbar puncture results excluded inflammation of the nervous system. In addition, the EEG performed identified a spike and slow wave patterns localized to the left hemisphere of the brain. These results indicate that the seizure originated from the lesion in the left parietal lobe of the patient.

Aphasia can either be an ictal or a post-ictal event. In the present report, the EEG showed spikes in the left tempo-central region, which correlated with the aphasia observed in the patient and the fact that the patient's consciousness level remained intact. The co-occurence of aphasia and electrographic seizure patterns in the present case indicated that the aphasia was a manifestation of an ictal event. Furthermore, the patient had complete language arrest but could follow instructions. Therefore, the patient was diagnosed with ictal Broca's aphasia, and regained a near baseline language function once their seizures were treated and their EEG results returned to normal.

Although, aphasia is a common symptom of neurological diseases, aphasic status epilepticus is a rare condition. An accurate conclusive diagnosis of aphasic status epilepticus will improve the patient's prognosis. EEG and other imaging techniques, including functional brain imaging, can help differentiate an aphasic seizure from aphasia resulting from other causes, including a stroke or migraine. In the present study, it was observed that EEGs were a simple and reliable method to diagnose aphasic seizures. Therefore, EEG recordings should be performed in all cases of unexplained aphasia, even without clinical evidence of seizures. 


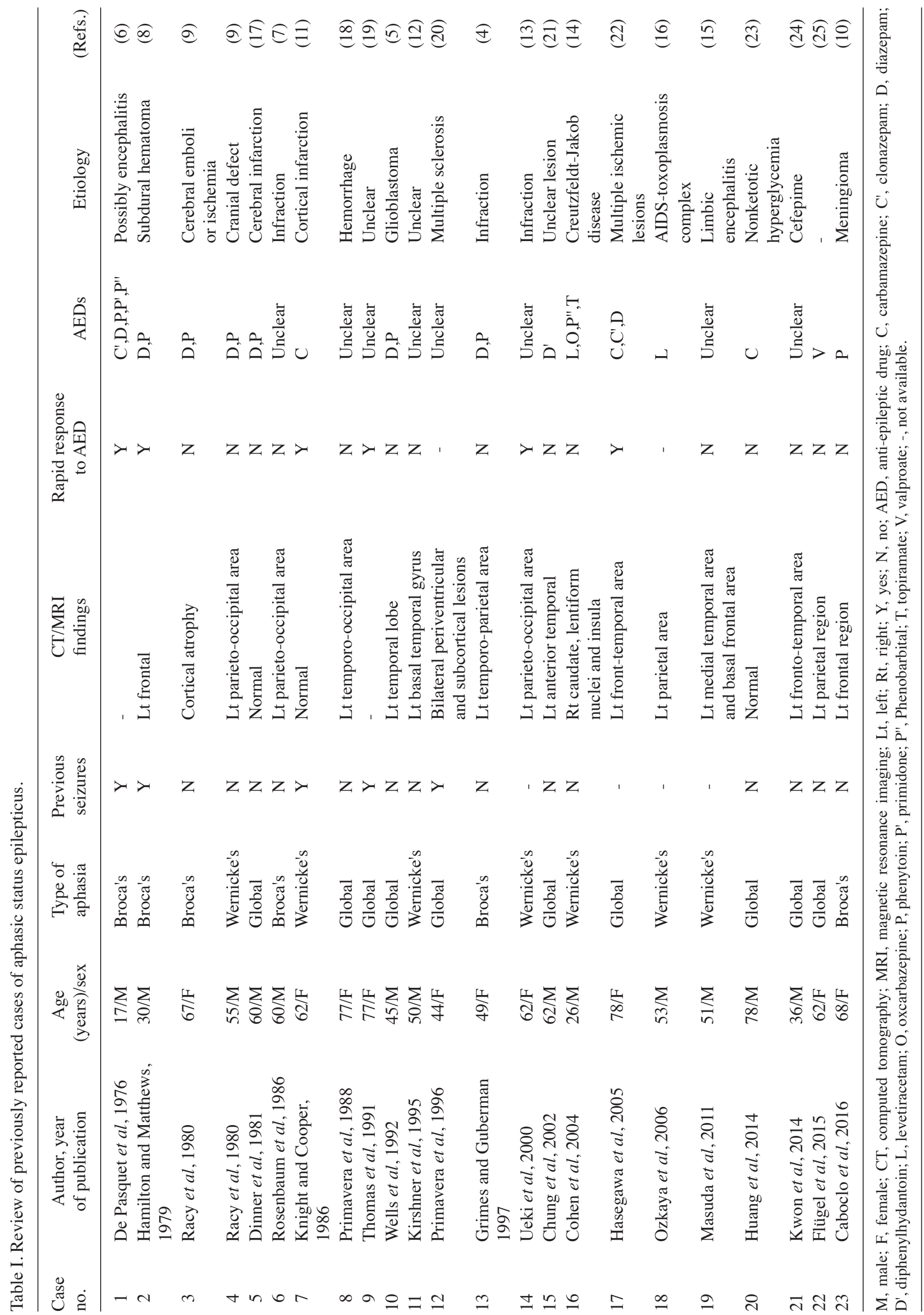




\section{Acknowledgements}

This manuscript has been edited and proofread by Medjaden Bioscience Limited.

\section{References}

1. Albert ML and Helm-Estabrooks N: Diagnosis and treatment of aphasia. Part I. JAMA 259: 1043-1047, 1988.

2. Kertesz A: Clinical forms of aphasia. Acta Neurochir Suppl (Wien) 56: 52-58, 1993.

3. Kanemoto K and Janz D: The temporal sequence of aura-sensations in patients with complex focal seizures with particular attention to ictal aphasia. J Neurol Neurosurg Psychiatry 52: $52-56,1989$.

4. Grimes DA and Guberman A: De novo aphasic status epilepticus. Epilepsia 38: 945-949, 1997.

5. Wells CR, Labar DR and Solomon GE: Aphasia as the sole manifestation of simple partial status epilepticus. Epilepsia 33 84-87, 1992.

6. De Pasquet EG, Gaudin ES, Bianchi A and De Mendilaharsu SA Prolonged and monosymptomatic dysphasic status epilepticus. Neurology 26: 244-247, 1976.

7. Rosenbaum DH, Siegel M, Barr WB and Rowan AJ: Epileptic aphasia. Neurology 36: 822-825, 1986.

8. Hamilton NG and Matthews T: Aphasia: The sole manifestation of focal status epilepticus. Neurology 29: 745-748, 1979.

9. Racy A, Osborn MA, Vern BA and Molinari GF: Epileptic aphasia. First onset of prolonged monosymptomatic status epilepticus in adults. Arch Neurol 37: 419-422, 1980.

10. Caboclo LO, Sanches PR, da Costa Neves RS and de Almeida Pimentel PC: De novo aphasic status epilepticus presenting with frontal periodic lateralized epileptiform discharges. Clin Neurophysiol 127: 973-975, 2016.

11. Knight RT and Cooper J: Status epilepticus manifesting as reversible Wernicke's aphasia. Epilepsia 27: 301-304, 1986.

12. Kirshner HS, Hughes T, Fakhoury T and Abou-Khalil B: Aphasia secondary to partial status epilepticus of the basal temporal language area. Neurology 45: 1616-1618, 1995.

13. Ueki Y, Terada K, Otsuka A, Kanda M and Akiguchi I: A case of non-convulsive status epilepticus worsened Wernicke's aphasia reversely. Rinsho Shinkeigaku 40: 339-343, 2000 (In Japanese).

14. Cohen D, Kutluay E, Edwards J, Peltier A and Beydoun A Sporadic Creutzfeldt-Jakob disease presenting with nonconvulsive status epilepticus. Epilepsy Behav 5: 792-796, 2004.
15. Masuda T, Kimura N, Nakamura K, Okazaki T, Arakawa R and Kumamoto T: A case of limbic encephalitis repeated aphasic status epilepticus with periodic lateralized epileptiform discharges. Rinsho Shinkeigaku 51: 135-140, 2011.

16. Ozkaya G, Kurne A, Unal S, Oguz KK, Karabudak R and Saygi S: Aphasic status epilepticus with periodic lateralized epileptiform discharges in a bilingual patient as a presenting sign of 'AIDS-toxoplasmosis complex'. Epilepsy Behav 9: 193-196, 2006.

17. Dinner DS, Lueders H, Lederman R and Gretter TE: Aphasic status epilepticus: A case report. Neurology 31: 888-891, 1981.

18. Primavera A, Bo GP and Venturi S: Aphasic status epilepticus. Eur Neurol 28: 255-257, 1988.

19. Thomas P, Kullmann B and Chatel M: Status epilepticus with aphasic manifestation. Rev Neurol (Paris) 147: 246-250, 1991 (In French).

20. Primavera A, Gianelli MV and Bandini F: Aphasic status epilepticus in multiple sclerosis. Eur Neurol 36: 374-377, 1996.

21. Chung PW, Seo DW, Kwon JC, Kim H and Na DL: Nonconvulsive status epilepticus presenting as a subacute progressive aphasia. Seizure 11: 449-454, 2002.

22. Hasegawa T, Shiga Y, Narikawa K, Jin K, Fujihara K, Takeda A and Itoyama Y: Periodic episodes of aphasia as an unusual manifestation of partial status epilepticus. J Clin Neurosci 12: 820-822, 2005

23. Huang LC, Ruge D, Tsai CL, Wu MN, Hsu CY, Lai CL and Liou LM: Isolated aphasic status epilepticus as initial presentation of nonketotic hyperglycemia. Clin EEG Neurosci 45: 126-128, 2014.

24. Kwon J, Choi JY and Bae EK: Cefepime-induced aphasic status epilepticus mimicking acute stroke. J Epilepsy Res 4: 85-87, 2014.

25. Flügel D, Kim OC, Felbecker A and Tettenborn B: De novo status epilepticus with isolated aphasia. Epilepsy Behav 49: 198-202, 2015.

26. Pedersen PM, Jorgensen HS, Nakayama H, Raaschou HO and Olsen TS: Aphasia in acute stroke: Incidence, determinants, and recovery. Ann Neurol 38: 659-666, 1995.

27. Perez ER and Davidoff V: Sign language in childhood epileptic aphasia (Landau-Kleffner syndrome). Dev Med Child Neurol 43: 739-744, 2001.

28. Krause-Titz UR, Warneke N, Freitag-Wolf S, Barth H and Mehdorn HM: Factors influencing the outcome (GOS) in reconstructive cranioplasty. Neurosurg Rev 39: 133-139, 2016. 